\title{
Evolution du réseau de surveillance de la qualité des sédiments portuaires (REPOM) pour la prise en compte des substances prioritaires européennes.
}

\section{Julie DROIT ${ }^{1}$, Jocelyn HILIOU ${ }^{2}$, Mohamed EL FADILI ${ }^{1}$, Marion MESSAGER ${ }^{1}$}

1. CEREMA eau, mer et fleuves, 155 rue Pierre Bouguer, BP 5, 29280 Plouzané, France.

julie.droit@cerema.fr ; mohamed.el-fadili@cerema.fr ; marion.messager@cerema.fr

2. Institut Universitaire Européen de la Mer (IUEM), Technopôle Brest-Iroise, rue Dumont d’Urville 29280 Plouzané, France.

\section{Résumé :}

Créé en 1997, le REPOM est un réseau de suivi national de la qualité des eaux et des sédiments des ports maritimes et estuariens mis en œuvre par les services en charge de la police des eaux littorales.

Depuis 2010, le programme de suivi dans l'eau a été suspendu au profit d'un développement du programme sédiments. Ainsi, le suivi de la qualité des sédiments a été complété par l'intégration des substances prioritaires de la Directive Cadre sur l'Eau (DCE).

En 2014, le REPOM a été intégré au réseau de surveillance de la Directive Cadre Stratégie Milieu Marin (DCSMM) pour la définition du bon état écologique des eaux marines au titre du descripteur 8 relatif aux contaminants.

Le protocole d'échantillonnage a été revu de façon à harmoniser les résultats et à ne suivre que la contamination récente des sédiments.

L'analyse des données de la "phase transitoire" 2010-2015, a permis de définir les niveaux de présence des substances prioritaires dans les sédiments portuaires, d'identifier les substances les plus fréquemment mesurées dans les sédiments, et de proposer des scenarii d'évolution du réseau pour les années à venir.

Mots-clés : Sédiments, Ports maritimes, Réseau de surveillance, Environnement côtier, Ecotoxicologie, Produits chimiques, Directives européennes, Contaminants prioritaires 


\section{Thème 6 - Gestion durable des zones littorales et estuariennes}

\section{Introduction}

Créé en 1997, le REPOM est un réseau de suivi national de la qualité des eaux et des sédiments des ports maritimes et estuariens mis en œuvre par les services en charge de la police des eaux littorales.

Depuis 2010, le programme de suivi dans l'eau a été suspendu au profit d'un développement du programme sédiments. Ainsi, le suivi de la qualité des sédiments a été complété par l'intégration des substances prioritaires de la Directive cadre sur l'eau (DCE).

Près d'une centaine de contaminants sont analysés au niveau de chaque point de surveillance.

Tableau 1. Groupes de contaminants suivis dans le cadre du REPOM.

\begin{tabular}{|c|c|}
\hline Groupes de contaminants & Contaminants \\
\hline $\begin{array}{l}\text { Contaminants réglementés dans le cadre des } \\
\text { dragages }\end{array}$ & Métaux lourds, HAP, PCB, TBT \\
\hline Organostaniques & $\begin{array}{l}\text { TBT et dérivés : DBT, } M B T \\
\text { Triphenylétain cation }\end{array}$ \\
\hline Organochlorés & $\begin{array}{l}(\alpha, \beta, \gamma) H C H, \text { aldrine, dieldrine, endrine, isodrine, } \\
H C B, D D T, \text { endosulfane }\end{array}$ \\
\hline Organophosphates & Trifluraline \\
\hline Phenols et dérivés & $\begin{array}{l}\text { Nonylphenol, octylphenol, pentachlorophenol, } \\
\text { tetrabromobisphenol-A }\end{array}$ \\
\hline Composés bromés & PBDE,$(\alpha, \beta, \gamma) H B C D$ (depuis 2014) \\
\hline Phtalates & DEHP \\
\hline Composés perfluorés & PFOS (depuis 2014) \\
\hline Dioxines et Furanes & 2,3,7,8-T4CDD, 2,3,7,8-T4CDF... (depuis 2014) \\
\hline
\end{tabular}

Le REPOM a été intégré en 2014 au réseau de surveillance de la Directive Cadre Stratégie Milieu Marin (DCSMM) pour la définition du bon état écologique des eaux marines au titre du descripteur 8 relatif aux contaminants.

L'analyse des données de la "phase transitoire" 2010-2015 a pour objectif d'améliorer les connaissances sur les concentrations des substances prioritaires dans les sédiments marins et estuariens et d'optimiser le réseau pour les années à venir, en proposant une liste des substances prioritaires les plus fréquemment mesurées dans les sédiments portuaires. 


\section{XVèmes Journées Nationales Génie Côtier - Génie Civil \\ La Rochelle, 29 au 31 mai 2018}

\section{Méthode}

Entre 2010 et 2014, 213 ports en France métropolitaine et en outre-mer ont été suivis chaque année, et pour certains sur plusieurs points de surveillance. Depuis 2015, un seul point de suivi a été retenu par port, sauf exceptions (enjeux locaux, configuration du bassin portuaire, concentrations mesurées lors de la phase transitoire...). Le nombre de points de surveillance a ainsi été réduit à 174 (pour 130 ports). De plus, la fréquence d'échantillonnage a été réduite à une fois tous les trois ans. Dans le cadre de la surveillance DCSMM, l'objectif est de pouvoir évaluer l'évolution temporelle de la contamination des sédiments, et donc de disposer de données pour les sédiments les plus récents. Le protocole de prélèvement a ainsi été revu de façon à ne prélever que la couche superficielle des sédiments et à harmoniser les pratiques. Les concentrations de contaminants prioritaires mesurées dans les sédiments de ces ports entre 2010 et 2015 ont été exploitées à partir de cette base de données.

Les contaminants réglementés pour les activités de dragage (métaux lourds, tributylétain, HAP, PCB) ont été exclus de cette étude.

Des seuils indicatifs de concentrations dans les sédiments ont été recherchés parmi les différentes valeurs seuils existantes pour les substances prioritaires :

- Qsed : Quality standard for sediment ou NQE sed : Normes de Qualité Environnementale pour les sédiments. Ces valeurs sont issues de la dérivation via la méthode d'équilibre de partage des Normes de qualité environnementale (NQE) de la DCE disponibles pour le compartiment eau (COMMISSION EUROPEENNE, 2005);

- PNEC : Predicted no effect concentration: Il s'agit d'un seuil écotoxicologique correspondant à la concentration d'une substance prédite sans effet pour un vecteur d'exposition donné ;

- EAC : Environmental Assessment Criteria. Ces valeurs sont utilisées dans le cadre de la Convention OSPAR pour la protection du milieu marin de l'Atlantique du Nord-Est (OSPAR, 2012).

\section{Résultats}

Les fréquences de quantification des contaminants ainsi que le dépassement des valeurs seuils indicatives ont été déterminés pour l'ensemble du littoral métropolitain ainsi que pour chacune des façades maritimes.

Le tableau 2 présente les valeurs seuils indicatives des substances prioritaires pour lesquelles des données sont disponibles : 


\section{Thème 6 - Gestion durable des zones littorales et estuariennes}

Tableau 2. Valeurs seuils indicatives déterminées à partir des données disponibles pour certaines substances prioritaires :

\begin{tabular}{|c|c|c|c|}
\hline Famille & Substance & $\begin{array}{l}\text { Valeur seuil } \\
\text { Indicative } \\
(\mu \mathrm{g} / \mathrm{kg})\end{array}$ & Source \\
\hline Organophosphorés & Trifluraline & 3140 & QSsed INERIS (2014) \\
\hline \multirow{4}{*}{ Composés bromés } & $\begin{array}{l}\text { PBDE } 209 \\
\text { (Polybromodiphényléther) }\end{array}$ & 310 & EAC OSPAR (2012) \\
\hline & OBDE (Octabromodiphényléther) & 5930 & QSsed INERIS (2012) \\
\hline & $\begin{array}{l}\text { PBDE (somme isomères 28, 47, 99, } \\
100,153,154)\end{array}$ & 6 & NQE sed Aquaref (2010) \\
\hline & HBCD (Hexabromocyclododécane) & 170 & QSsed INERIS (2012) \\
\hline \multirow{5}{*}{ Phénols et dérivés } & Nonylphenols & 39 & PNEC Ineris (2014) \\
\hline & Para-ter t-octylphenol ramifiés & 24 & NQE sed Aquaref (2010) \\
\hline & Pentachlorophenol & 170 & NQE sed Aquaref (2010) \\
\hline & TBBP-A (Tétrabromobisphénol-A) & 410 & PNEC/Qsed Ineris (2014) \\
\hline & Octylphenol & 24 & NQE sed Aquaref (2010) \\
\hline \multirow{5}{*}{ Organochlorés } & HCB (hexachlorobenzene) & 85 & NQE sed Aquaref (2011) \\
\hline & $\begin{array}{l}\text { DDT totaux } \\
\text { (dichlorodiphényltrichloroéthane) }\end{array}$ & 83,6 & NQE sed Aquaref (2016) \\
\hline & Dieldrine & 3 & NQE sed Aquaref (2010) \\
\hline & Aldrine & 10 & NQE sed Aquaref (2010) \\
\hline & Isodrine & 11 & NQE sed Aquaref (2010) \\
\hline Phtalates & DEHP (Di(2éthylhexyl) Phtalate) & 4720 & NQE sed Aquaref (2009) \\
\hline $\begin{array}{l}\text { Composés } \\
\text { perfluorés }\end{array}$ & $\begin{array}{l}\text { PFOS (acide } \\
\text { perfluorooctanesulfonique) }\end{array}$ & 67 & PNEC/Qsed Ineris (2014) \\
\hline
\end{tabular}

Pour certaines substances, aucune VSI n'a pu être établie en raison d'une absence de données. Les substances concernées sont: le méthyl-mercure, les $(\alpha, \beta, x) \operatorname{HBCD}$, le triphénylétain, le delta-HCH, les PBDE 206 et 207, ainsi que les dioxines et furanes.

Le tableau 3 présente les substances les plus fréquemment quantifiées pour tous les ports métropolitains suivis dans le cadre du REPOM (à l'exclusion des contaminants réglementés pour les activités de dragage): 


\section{XVèmes Journées Nationales Génie Côtier - Génie Civil \\ La Rochelle, 29 au 31 mai 2018}

Tableau 3. Substances prioritaires les plus fréquemment quantifiées dans les sédiments des ports suivis dans le cadre du REPOM entre 2010 et 2015 (hors contaminants réglementés dans le cadre des dragages).

\begin{tabular}{|l|l|l|l|}
\hline Substances & $\begin{array}{l}\text { Fréquence de } \\
\text { quantification } \\
\text { (en\%) }\end{array}$ & $\begin{array}{l}\text { Fréquence des résultats } \\
\text { supérieurs à la valeur seuil } \\
\text { (en\%) }\end{array}$ & $\begin{array}{l}\text { Nombre } \\
\text { d'analyses }\end{array}$ \\
\hline Hexabromocyclododécane (HBCDD) & 100 & 0 & 16 \\
\hline Diéthylhexylphtalate (DEHP) & 82 & 3 & 1043 \\
\hline Dibutylétain cation (DBT) & 82 & $/$ & 1182 \\
\hline Dioxines et furanes & 74 & $/$ & 476 \\
\hline Monobutylétain (MBT) & 74 & $/$ & 1163 \\
\hline $\begin{array}{l}\text { Dochlorodiphényl dichloroéthane op' } \\
\text { (p, p'DDT ou 4, 4'DDT) }\end{array}$ & 59 & 5 & 304 \\
\hline Polybromodiphényléther 209 (PBDE 209) & 54 & 0 & 61 \\
\hline
\end{tabular}

Parmi ces substances l'Hexabromocyclododécane (HBCDD) présente un faible nombre d'analyses. L'analyse des données 2016 et 2017 permettra de déterminer son degré de présence dans les sédiments portuaires.

Le DEHP, le DBT, et le MBT ont fait l'objet de plus de 1000 analyses entre 2010 et 2015 et sont quantifiés dans plus de $70 \%$ des analyses.

Le tableau 4 présente les substances peu ou pas quantifiées dans les sédiments des ports métropolitains suivis dans le cadre du REPOM. Une analyse du respect des limites de quantification analytiques préconisées dans le cadre du cahier des charges du REPOM (MEDDE - CEREMA, 2015) a également été menée.

Tableau 4. Substances prioritaires peu (<3\%) ou pas quantifiées dans les sédiments des ports suivis dans le cadre du REPOM entre 2010 et 2015 et respect des limites de quantification analytiques.

\begin{tabular}{|l|l|l|}
\hline Substances & Nombre d'analyses & \% non respect des $\mathbf{L Q}$ \\
\hline Endosulfan & 263 & $1,10 \%$ \\
\hline Sulfate endosulfan & 492 & $8,70 \%$ \\
\hline Aldrine & 1145 & $36,90 \%$ \\
\hline Isodrine & 1046 & $27,00 \%$ \\
\hline Alpha HCH & 1131 & $30,10 \%$ \\
\hline Trifluraline & 1139 & $55,20 \%$ \\
\hline
\end{tabular}

D'après les données 2010-2015, l'endosulfan et le sulfate endosulfan sont très faiblement quantifiés dans les sédiments portuaires. 


\section{Thème 6 - Gestion durable des zones littorales et estuariennes}

Pour les autres substances, le faible pourcentage de quantification peut être dû à des limites de quantification analytique trop élevées. Les données 2016 et 2017 seront nécessaires pour qualifier le degré de présence de ces contaminants dans les sédiments portuaires.

\section{Conclusions}

Le bilan de la phase transitoire du REPOM (2010-2015) a permis d'identifier un certain nombre de recommandations pour la surveillance des substances prioritaires de la DCE et de la DCSMM dans les sédiments portuaires:

- Les substances peu ou pas quantifiées sur l'ensemble du littoral ou sur une ou plusieurs façades maritimes pourraient ne plus être suivies dans les zones concernées : endosulfan, sulfate endsulfan, alpha-HCH...

- La surveillance des substances quantifiées sur plus de 20\% des échantillons et dont les concentrations peuvent dépasser les valeurs seuils indicatives pourrait être renforcée: DEHP, PBDE 209, DDT et dérivés, et nonylphénol.

L'analyse des données 2016 et 2017 permettra d'affiner ces premières conclusions et d'optimiser le réseau pour les années à venir.

\section{Références}

MEDDE - CEREMA. (2015). REPOM. Cahier des charges pour la mise en œuvre du programme dès 2015.

COMMISSION EUROPEENNE. (2005). Common implementation strategy for the water framework directive. Environmental Quality Standards. Substance data Sheet. OSPAR (2012). General advice. Review of environmental assessment criteria or equivalents. 